\title{
Battling Biofilm Forming Nosocomial Pathogens Using Chitosan and Pluronic F127
}

\author{
Doaa Eid $^{1}(\mathbb{D})$, Ossama M. Sayed ${ }^{2}(\mathbb{D})$, Walaa G. Hozayen ${ }^{3}$ iD and Ahmed F. Azmy ${ }^{4 *}$ (D) \\ ${ }^{1}$ Biotechnology and Life Sciences Department, Faculty of Postgraduate Studies for Advanced Sciences (PSAS), \\ Beni-Suef University, Egypt. ${ }^{2}$ Pharmaceutics and Industrial Pharmacy Department, Faculty of Pharmacy, Beni- \\ Suef University, 62511, Beni-Suef, Egypt. ${ }^{3}$ Biochemistry Division, Chemistry Department, Faculty of Science, \\ Beni-Suef University, Egypt. ${ }^{4}$ Microbiology and Immunology Department, Faculty of Pharmacy, Beni-Suef \\ University, 62511, Beni-Suef, Egypt.
}

\begin{abstract}
Biofilm represents a potential strut in bacterial treatment failure. It has a dual action; it affords microbial resistance against antibiotics and facilitate transmission of pathogenic bacteria. Nosocomial bacteria pose a serious problem in healthcare units; it prolongs patient hospital stay and increases the mortality rates beside other awful economical effect. This study was planned for targeting nosocomial bacterial biofilm using natural and biologically safe compounds like Chitosan and/or Pluronic F127. Ninety-five isolates were recovered from 107 nosocomial clinical samples. Different bacterial and fungal species were detected, from which Klebsiella pneumonia (23\%), Pseudomonas aeruginosa (19\%), Acinetobacter baumannii (18\%) and E.coli (17\%) were the predominate organisms. Pseudomonas aeruginosa, Acinetobacter baumanni and Klebsiella pneumonia were the abundant antibiotic resistant strains with multi-resistance pattern of $72 \%, 65 \%$ and $59 \%$, respectively. A significant percentage of these isolates were strong biofilm forming. Herein, we investigate the effect of Chitosan and Pluronic F127 alone and in combination with each other against biofilm production. Chitosan show variable degree of biofilm inhibition, while Pluronic F127 was able to retard biofilm formation by $80 \%$ to $90 \%$ in most strain. There is no significant difference $(P<0.05)$ between Pluronic F127 alone and its effect in combination with Chitosan.
\end{abstract}

Keywords: Nosocomial infection, Biofilm, Chitosan, Pluronic F127

*Correspondence: ahmed.abdelaziz@pharm.bsu.edu.eg; +2-01004457502

(Received: July 07, 2020; accepted: September 04, 2020)

Citation: Eid D, Sayed OM, Hozayen WG, Azmy AF. Battling Biofilm Forming Nosocomial Pathogens Using Chitosan and Pluronic F127. J Pure Appl Microbiol. 2020;14(3):1893-1903. doi: 10.22207/JPAM.14.3.28

(C) The Author(s) 2020. Open Access. This article is distributed under the terms of the Creative Commons Attribution 4.0 International License which permits unrestricted use, sharing, distribution, and reproduction in any medium, provided you give appropriate credit to the original author(s) and the source, provide a link to the Creative Commons license, and indicate if changes were made. 


\section{INTRODUCTION}

Nosocomial infection is a major health care problem. It causes a large percent of morbidity and mortality, one from ten patients is affected by this type of infection ${ }^{1}$. Nosocomial infection result in prolonged hospital stay with increased healthcare costs $^{2}$. These infections are mainly caused by multi or extensive drug resistant organisms like Methicillin-resistant Staphylococcus aureus (MRSA) and extended spectrum $\beta$-lactamases (ESBL). Scarcity in discovery of a new antibiotic generation worsen the problem, so the prevention is a suitable approach for decreasing this shocking infection².

According to National Health Institute (NIH) $65-80 \%$ of chronic microbial infections are related to biofilm forming microorganisms ${ }^{3}$. The biofilm infection may be device associated or non-device associated, upon detachment from the surface the microorganism releases hydrolytic enzymes enabling them to colonize a new area ${ }^{4}$. Colonization may occur in a sensitive and vital places in human body like endocardium, heart valves, lungs and joints resulting in dangerous and life-threatening infections ${ }^{5}$. The biofilm may be performed on or within medical devices such as venous catheter, contact lenses and prosthetic heart valves by one microorganism or mixed infections according to device type and duration. Also, biofilm down side infection treatment as it decreases antibiotic penetration and allow exchange of resistance plasmids between stains in biofilm matrix ${ }^{6}$. Resistance in biofilm forming bacteria is significantly higher than the planktonic cells ${ }^{7}$. Therefore, it is so difficult to be eradicated and removed ${ }^{8}$.

For management of these infections a new strategy should be evolved. Use of antibiofilm compounds is an interesting one, it will decrease rate of transmission by decreasing attachment and colonization or used in combination with other antibiotics to enhance its activity 9 . Different types of compounds are used as antibiofilm agents like sub MIC concentration of antibiotics ${ }^{10}$, antimicrobial peptides ${ }^{11}$, enzymes ${ }^{12,13}$, or quorum sensing inhibitors like $\mathrm{N}$-acetyl homoserine ${ }^{14}$.

Chitosan is a natural biodegradable and biocompatible compound, has antibacterial activity in high concentration and potent antibiofilm activity at lower concentration. Molecular weight and acetylation degree affect its potency as antimicrobial agent ${ }^{15}$. Being as positively charged cations, chitosan can act in 3 different ways: by interaction with negatively charged microbial cells; interact with microbial DNA; or chelating important metals required for metalloprotein enzymes $^{16}$.

Pluronic F127 is a synthetic non-ionic surfactant with amphiphilic properties. It is a copolymer of hydrophilic poly(ethylene oxide) and hydrophobic poly(propylene oxide). Due to their amphiphilic characters, Pluronic has an excellent surfactant property ${ }^{17}$. Combination of chitosan and pluronic acid were mixed together in a nanoparticle form for delivery of anticancer drugs with less side effect ${ }^{18,19}$. The combination is also used for preparation of different pharmaceutical dosage forms $\mathrm{s}^{20,21}$

The aim of this study is to evaluate efficacy of chitosan and pluronic F127 alone and in combination with each other against biofilm forming nosocomial pathogens as a new tactic to retard their transmission and resistance.

\section{MATERIAL AND METHOD \\ Sample Isolation}

Different biological samples (urine, sputum, endotracheal secretion and blood) were collected from 107 patients between May 2017 and June 2018. All patients admitted to hospital for at least 3 days without previous signs or symptoms of previous infection. All samples were isolated from patients in ICU and neonatal ICU following ethical consideration.

Strains were isolated and purified using different types of media (Blood agar and MacConkey agar). All isolates were stored in glycerol broth at $-80^{\circ} \mathrm{C}$ till further analysis.

\section{Bacterial Identification and Antibiotic Sensitivity}

Bacterial isolates were primarily identified by Gram stain and biochemical reactions (catalase, oxidase, motility and triple sugar iron agar). Full identification was performed by VITEK2 system (BioMérieux, USA). Antibiotic sensitivity test was done by disc diffusion method according to CLSI guidelines $^{22}$ using Muller-Hinton agar and Brain Heart infusion agar. The antibiotic discs used were

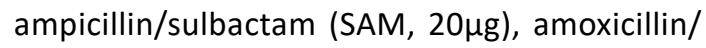




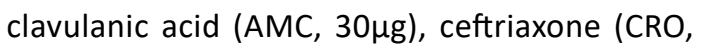
$30 \mu \mathrm{g})$, piperacillin/tazobactam (TZP, 110 $\mathrm{g}$ ),

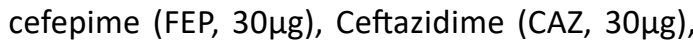

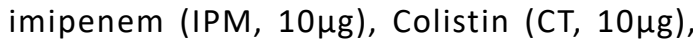

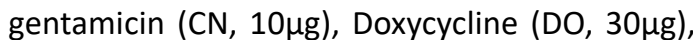

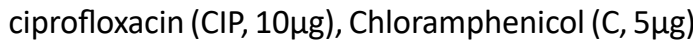
and Vancomycin (VA, $5 \mu \mathrm{g})$. Sensitivity pattern of Candida isolates is not determined.

\section{Biofilm Formation Assay of Isolated Pathogens}

A previously isolated pure colony was resuspended in $5 \mathrm{~mL}$ of tryptone soya broth supplemented with $1 \%$ glucose, incubated at $37^{\circ} \mathrm{C}$ or $30^{\circ} \mathrm{C}$ for $48 \mathrm{hrs}$ for bacteria and Candida, respectively. Twenty microliters of overnight culture were diluted in $180 \mu \mathrm{L}$ of the above media in 96 well sterile microplate and incubated at $37^{\circ} \mathrm{C}$ or $30^{\circ} \mathrm{C}$ for $48 \mathrm{hrs}$. After incubation the growth was discarded, and the plates were washed three times with phosphate buffered saline $\mathrm{pH} 7.5$ to remove non-adherent cells. The plates were dried in the oven at $65^{\circ} \mathrm{C}$ and stained with $200 \mu \mathrm{L}$ of $1 \%$ crystal violet solution for $15 \mathrm{~min}$. the plates were washed gently under running water and dried. A solution of $1 \%$ acetic acid is used to retain adsorbed C.V stain for $15 \mathrm{~min}$. and measured spectrophotometrically at $600 \mathrm{~nm}$ by microplate reader (Tecan SunRise/ USA). The strains were classified as weak, moderate or strong biofilm forming bacteria according to classification of Stepanovic et al. ${ }^{23}$ The experiments were done with six replicates in three independent experiments.

Effect of Chitosan and Pluronic Acid on Biofilm Formation

Chitosan was dissolved in $1 \%$ acetic acid solution to a final concentration of $10 \mathrm{mg} / \mathrm{mL}$. The solution was stirred overnight at $50^{\circ} \mathrm{C}$ to ensure complete dissolution, $\mathrm{pH}$ was raised to $5.8-6.0$ by $1 \mathrm{~N} \mathrm{NaOH}$ and sterilized by $0.2 \mu \mathrm{m}$ microbial filter (Sartorius, Germany). Pluronic F127 was dissolved at a concentration of $10 \mathrm{mg} / \mathrm{mL}$ in distilled water at $\mathrm{pH}$ of $7 \pm 0.2$. Bacterial overnight culture was diluted in TSB amended with $1 \%$ glucose to a concentration of $10^{8} \mathrm{CFU} / \mathrm{mL}$ containing different concentrations of Chitosan and/or Pluronic F127 $(5,2.5,1.25,0.625 \mathrm{mg} / \mathrm{ml})$. In a flat bottom microtiter plate, a $200 \mu \mathrm{L}$ of the above solution was added and incubated at $37^{\circ} \mathrm{C}$ or $30^{\circ} \mathrm{C}$ for $48 \mathrm{hr}$. Extent of biofilm inhibition occurred by Chitosan and/or Pluronic F127 is detected by C.V method. Percentage of inhibition is calculated by the following equation by Pierce et al. ${ }^{24}$ Inhibition \%= 100- (OD sample $\left./ O D_{\text {control }}\right) x$ 100

\section{Scanning Electron Microscope for Biofilm Inhibition Assay}

Ten microliter of 0.5 MacFarland solution of diluted overnight bacterial culture was placed in the center of Sterile Millipore membrane filter $(0.22 \mu \mathrm{m}, 47 \mathrm{~mm}$ diameter, mixed cellulose esters (MCE) membrane) (Merck, USA). The filter was loaded on tryptone soya agar plates (TSA) amended with $2.5 \mathrm{mg} / \mathrm{mL}$ of Chitosan, Pluronic F127 and combination of Chitosan and Pluronic F127. The plates were incubated at $37^{\circ} \mathrm{C}$ for 24 hr. The membrane filters were gently removed, fixed in $3 \%$ glutaraldehyde for $30 \mathrm{~min}$. The filters were washed 3 times with PBS each for $10 \mathrm{~min}$. The membranes were gradually dehydrated with gradient conc of ethyl alcohol $(50 \%, 60 \%$, $70 \%, 80 \%, 90 \%, 95 \%, 100 \%)$, then final chemical dehydration with hexamethyldisilazane. The coupons were coated with gold, and then examined with JSM-6510 (JEOL, Japan) at a voltage of $30 \mathrm{kV}$ and magnifications at $\times 5000$ to $\times 15000^{25}$. Statistical Analysis

Data were expressed as mean \pm standard error of mean (SEM). Statistical analysis was performed using statistical package for social sciences (SPSS) computer software (version 22), IBM software, USA. One-way ANOVA test was used to evaluate significance between groups followed by tukey posthoc analysis for pairwise analysis. Differences were considered statistically significant at $p<0.05$.

\section{RESULTS}

\section{Bacterial Isolation and Identification}

Out of 107 clinical samples taken, 95 samples were positive for bacterial or fungal culture. Large number of isolated strains were found in sputum (30 strains) and endotracheal intubation (28 strains). Urine samples were positive for 27 specimens while blood gives only 10 isolates (Table 1 ).

The top four species were Klebsiella pneumoniae (23\%), Pseudomonas aeruginosa (19\%), Acinetobacter baumannii (18\%) and E.coli $(17 \%)$. Gram +ve bacteria also present 
in a significant percentage represented by Staphylococcus aureus and Staphylococcus haemolyticus with $8 \%$ and $4 \%$ respectively. Other Gram-negative bacteria like Proteus spp. and Enterobacter spp. were found in a small frequency ( $3 \%$ and $2 \%$ consequently). Fungal infection with Candida albicans and Candida tropicalis were also reported with a lower incidence at 3 and $2 \%$ respectively. (Table 1 )

\section{Antibiotic Sensitivity Testing}

All Gram-positive strains were sensitive to Vancomycin, while all Gram-negative strains were sensitive to Colistin. Ten isolates (45\%) of Klebsiella, seven isolates (41\%) of Acinetobacter and six isolates (33\%) of Pseudomonas show resistance against all tested antibiotics. Four strains of E.coli and one isolate of Staphylococcus haemolyticus, Enterobacter spp., and Proteus spp. were resistant to nine of tested antibiotics. Overall, resistance to the Piperacillin/tazobactam and Imipenem were found to be much lower. (Table 2) Biofilm Formation Assay of Isolated Pathogen

Thirty-five isolates show no biofilm activity, while 26 and 24 isolates show weak and moderate biofilm forming ability, respectively. Only 10 isolates were strong biofilm producer and they were distributed as one strain of St. haemolyticus, one strain Candid tropicalis, three strains of Klebsiella pneumonia, two strains of Acinetobacter baumannii and three strains of Pseudomonas aeruginosa. (Fig. 1).

\section{Effect of Chitosan and Pluronic F127 on Biofilm Formation}

All isolated strains were significantly reduced to different levels by chitosan at the concentration used $2.5 \mathrm{mg} / \mathrm{mL}$. The most affected strain was the extensively drug resistant Pseudomonas aeruginosa (XDR) strain, it was decreased by $81 \%$ of initial biofilm formed in planktonic cells. Multi- and extensive drug resistant Klebsiella pneumonia strains (MDR and XDR) and Acinetobacter baumannii were inhibited by $25-35 \%$. Also, chitosan diminished biofilm of Pseudomonas aeruginosa (MDR), Staphylococcus haemolyticus and Candida albicans by 33\%, 51\% and $58 \%$ respectively. (Fig. 2 )

Pluronic F127 has a more potent effect than Chitosan in prevention of biofilm creation at concentration used $1.25 \mathrm{mg} / \mathrm{mL}$. It inhibits all Pseudomonas and Klebsiella spp. by more than $80 \%$. It also prohibits biofilm production in other strains of Staphylococcus haemolyticus, Acinetobacter baumannii and Candida albicans by $69 \%, 43 \%$ and $59 \%$ respectively. Statistical analysis shows significant difference $(P<0.05)$ in biofilm inhibition between Pluronic F127 and Chitosan in Klebsiella pneumonia (XDR, MDR) and Pseudomonas aeruginosa (MDR). Other strains show no significant difference between Pluronic F127 and Chitosan. overall, all strains show no significant difference between Pluronic F127 and Mixture of both materials. (Fig. 2)

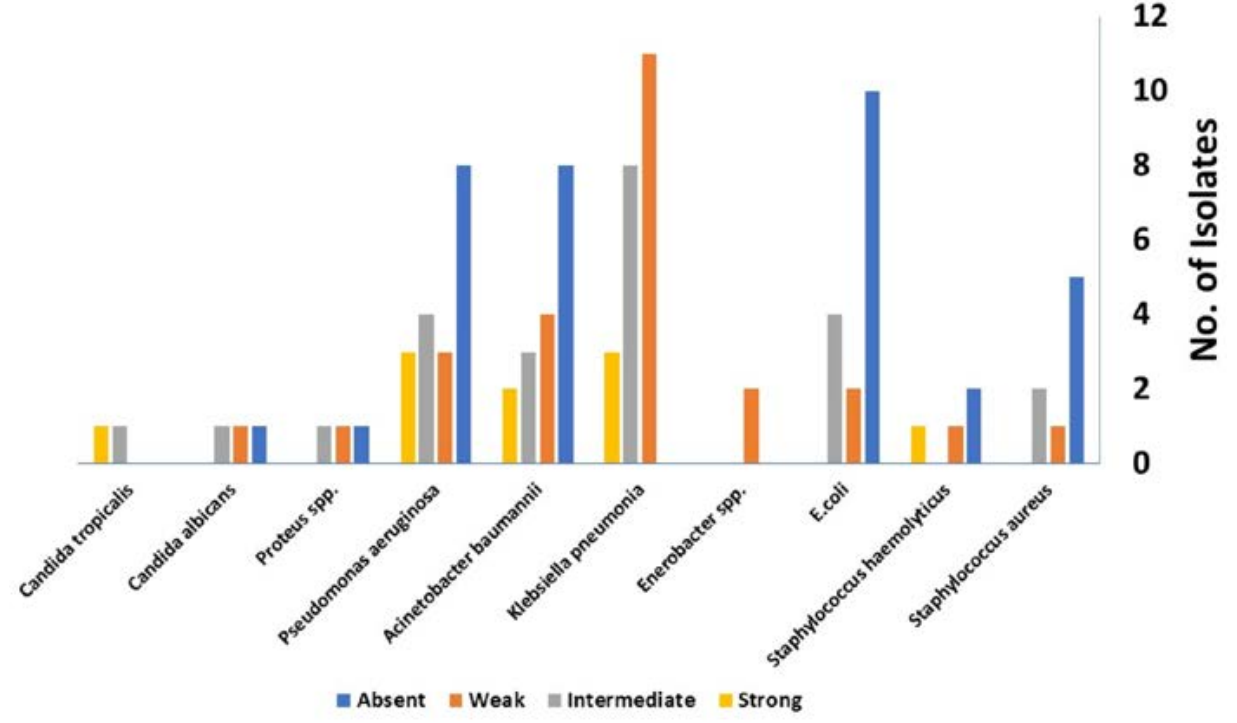

Fig. 1. Biofilm forming ability of clinical isolates recovered from different nosocomial clinical samples. 

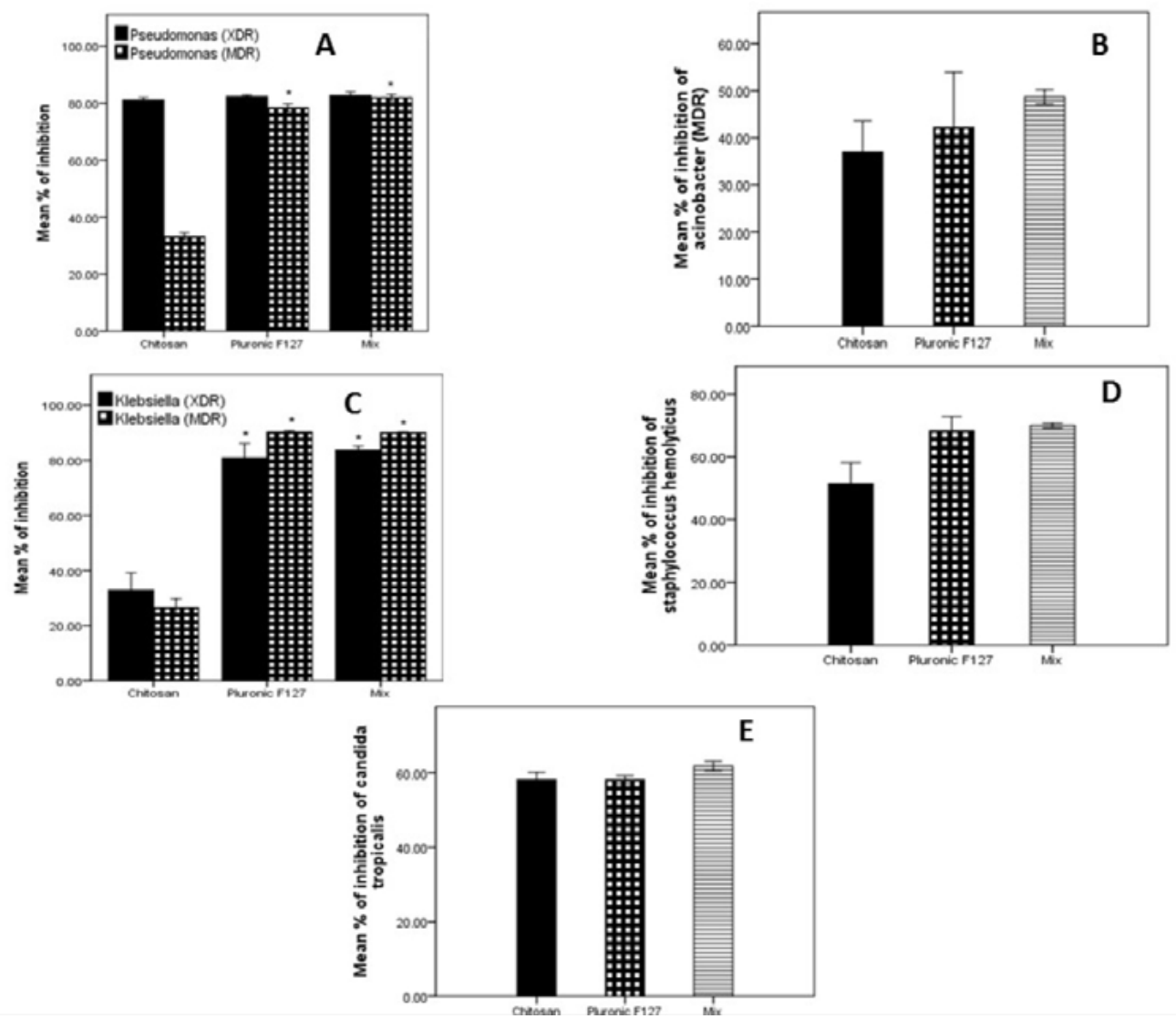

Fig. 2. Effect of Chitosan and Pluronic F127 on biofilm formation on (a) multi drug resistant Pseudomonas aeruginosa (MDR) and extensive drug resistant Pseudomonas aeruginosa (XDR) (b) Staphylococcus haemolyticus (c) multi drug resistant Klebsiella pneumoniae (MDR) and extensive drug resistant Klebsiella pneumoniae (XDR) (d) extensive drug resistant Acinetobacter baumannii (e) Candida tropicalis.

Table 1. Distribution of Nosocomial bacteria isolated from different clinical samples

\begin{tabular}{lccccc}
\hline \multirow{2}{*}{ Organism } & Number & \multicolumn{4}{c}{ lsolation specimen } \\
\cline { 3 - 6 } & & Urine & Sputum & $\begin{array}{c}\text { endotracheal } \\
\text { secretion }\end{array}$ & Blood \\
\hline Staphylococcus aureus & 8 & 1 & 3 & 3 & 1 \\
Staphylococcus haemolyticus & 4 & 1 & 2 & 1 & - \\
E.coli & 16 & 13 & 1 & 1 & 1 \\
Enerobacter spp. & 2 & 2 & - & - & - \\
Klebsiella pneumonia & 22 & 2 & 10 & 7 & 3 \\
Acinetobacter baumannii & 17 & - & 6 & 9 & 2 \\
Pseudomonas aeruginosa & 18 & 4 & 6 & 7 & 1 \\
Proteus spp. & 3 & 2 & 1 & - & - \\
Candida albicans & 3 & 1 & 1 & - & 1 \\
Candida tropicalis & 2 & 1 & - & - & 10 \\
Total No. & 95 & 27 & 30 & 28 & \\
\hline
\end{tabular}




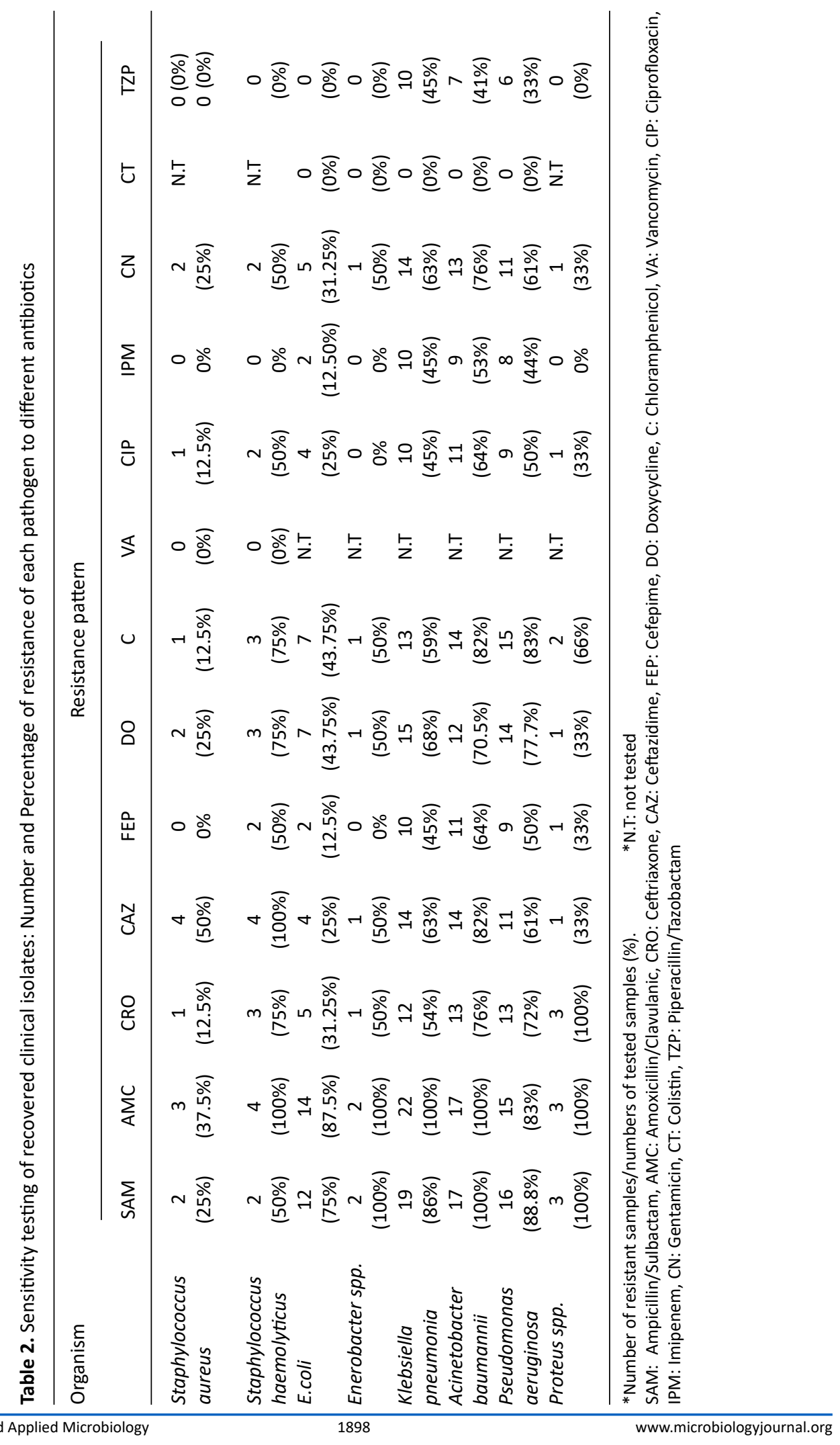


Table 3. Multi-drug resistance pattern of isolated nosocomial bacterial strains

\begin{tabular}{lccccc}
\hline \multirow{2}{*}{ Strain } & \multicolumn{5}{c}{ Degree of resistance (No of antibiotic) } \\
\cline { 2 - 6 } & $\geq 11$ & $8-10$ & $5-7$ & $2-4$ & $\leq 1$ \\
\hline Staphylococcus aureus $(\mathrm{n}=8)$ & $0(0 \%)$ & $1(12.5 \%)$ & $1(12.5 \%)$ & $2(25 \%)$ & $4(50 \%)$ \\
Staphylococcus haemolyticus $(\mathrm{n}=4)$ & $0(0 \%)$ & $2(50 \%)$ & $0(0 \%)$ & $2(50 \%)$ & $0(0 \%)$ \\
E.coli $(\mathrm{n}=16)$ & $0(0 \%)$ & $2(12.5 \%)$ & $3(19 \%)$ & $9(56 \%)$ & $2(12.5 \%)$ \\
Enerobacter spp. $(\mathrm{n}=2)$ & $0(0 \%)$ & $0(0 \%)$ & $1(50 \%)$ & $1(50 \%)$ & $0(0 \%)$ \\
Klebsiella pneumonia $(\mathrm{n}=22)$ & $10(45 \%)$ & $0(0 \%)$ & $3(14 \%)$ & $6(27 \%)$ & $3(14 \%)$ \\
Acinetobacter baumannii $(\mathrm{n}=17)$ & $7(41 \%)$ & $3(18 \%)$ & $1(6 \%)$ & $1(6 \%)$ & $5(29 \%)$ \\
Pseudomonas aeruginosa $(\mathrm{n}=18)$ & $6(33 \%)$ & $3(17 \%)$ & $4(22 \%)$ & $1(6 \%)$ & $4(22 \%)$ \\
Proteus spp. $(\mathrm{n}=3)$ & $0(0 \%)$ & $1(33 \%)$ & $0(0 \%)$ & $2(66 \%)$ & $0(0 \%)$ \\
\hline
\end{tabular}

\section{Electron Microscopic Image of Bacterial Strains}

Biofilm inhibition by chitosan and/or pluronic acid were also confirmed by imaging with scanning electron microscope (SEM). Untreated strains show a high percentage of exopolysaccharide matrix with increased aggregation of cells in thick multicellular pattern. While, treated cells with chitosan and/or pluronic acid show a well isolated microcolonies with limited or no exopolysaccharide materials formed. (Fig. 3,4)

\section{DISCUSSION}

Most nosocomial infections are attributed to organisms with considerable degree of antibiotic resistance. This leads to increased demand on discovering new types of antibiotics with decreased resistance. Targeting bacterial biofilm is another strategy to reduce their transmission and increase efficacy of antibiotics.

The present study shows the high incidence of nosocomial infection among clinical samples, it is approximately $88 \%$. This remarkable higher level may be attributed to
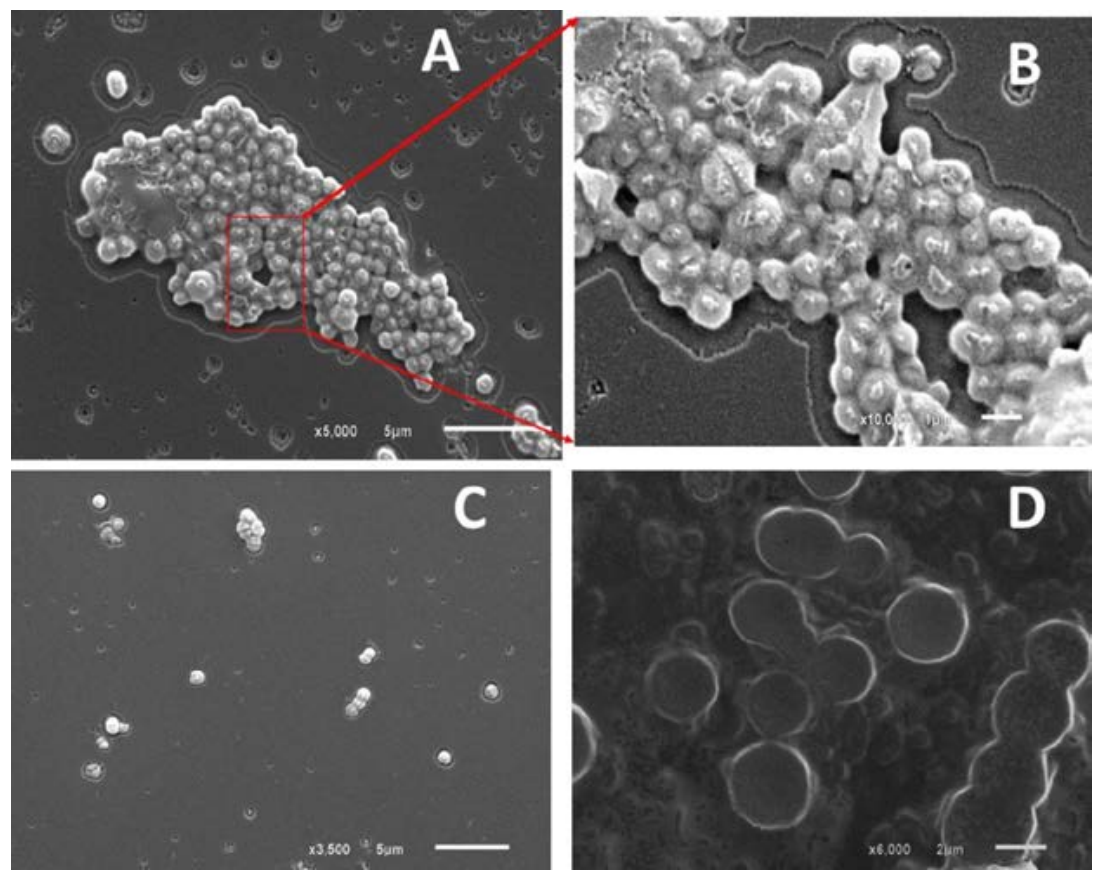

Fig. 3. Biofilm formation in Staphylococcus haemolyticus (a) Planktonic cell (b) enlargement of red box view of planktonic cell (c) in presence of Pluronic F127 (d) in presence of Chitosan. 


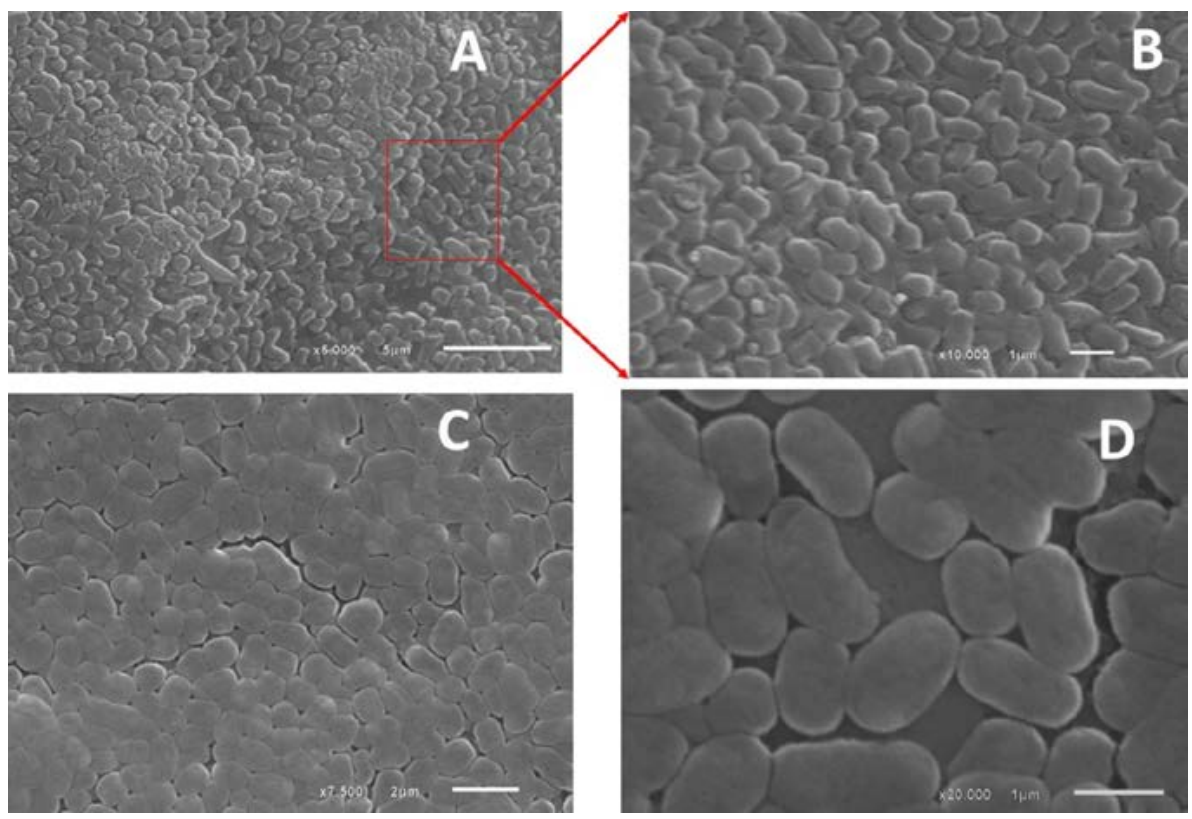

Fig. 4. Biofilm formation in Klebsiella pneumoniae (a) Planktonic cell (b) enlargement of red box view of planktonic cell (c) in presence of Pluronic F127 (d) in presence of Chitosan.

misuse of prescribed antibiotics and absence of disinfection policy in these hospitals This finding is in accordance with Mama et al who found a similar result (87\%) at Jimma hospital ${ }^{26}$. Also, Dessie and his colleagues reported a high incidence level $(84 \%)$ in Addis Ababa hospital ${ }^{27}$. Other researchers recorded a lower level $66 \%{ }^{28}$ at Gondor University hospital and $70 \%$ at Ethiopia ${ }^{29}$.

Klebsiella pneumonia, Pseudomonas aeruginosa, Acinetobacter baumannii and E.coli were the predominate isolates with a low incidence of Staphylococcus aureus infection. This results is in agreement with Matta et al. who reported that Pseudomonas aeruginosa (12\%), Klebsiella pneumoniae (6.2\%) and Acinetobacter baumannii (3.1\%) were the paramount isolates recovered from 258 patients in Lebanon ${ }^{30}$. Another study by Peters et al. accounted for eighty-five percent of hospital-acquired infection were Acinetobacter baumanii (28\%), Klebsiella pneumoniae (25\%), Pseudomonas aeruginosa (21\%), Escherichia coli (9\%) and Serratia marcescens (3\%) $)^{31}$. On contrary, Sserwadda and his colleagues reported that Klebsiella pneumonia and Staphylococcus aureus were the most frequently isolated strains in Kawolo general hospital, Uganda ${ }^{32}$. Other researchers in a
Tertiary Hospital of northern Tanzania mentioned that Staphylococcus aureus was the most common isolated microorganism followed by Enterococcus and other coliform ${ }^{33}$.

Vancomycin was the most sensitive antibiotic against all isolated Staphylococcus strains. Also, there is no detectable resistance to Colistin in isolated Gram-negative bacteria. The antibiotic with least resistance was found to be against Piperacillin/Tazobactam, Imipenem and Meropenem. Large percentage of Klebsiella pneumonia, Acinetobacter baumannii and Pseudomonas aeruginosa were extensive drug resistant strains, they were resistant to twelve antibiotics from different classes. A similar finding was presented by Mauldin et al. who reported that fifty percent of hospital acquired infections were multi-drug resistant (MDR) ${ }^{34}$.

In our study Klebsiella pneumonia, Pseudomonas aeruginosa and Acinetobacter baumannii were potent biofilm producer while other types like Staphylococcus and Candida spp show weak to moderate biofilm forming ability. These results coincide with Mulla et al. who demonstrated that Acinetobacter, Pseudomonas, Klebsiella, Staphylococcus spp. were the main cause of infection in indwelling devices due to 
biofilm formation ${ }^{35}$. Also, Singhai et al. show that Klebsiella pneumonia represents the most abundant biofilm forming nosocomial microbe with multi-resistant pattern and extended $\beta$-lactamase producer ${ }^{36}$. Other researchers show relationship between microbial resistance and biofilm formation ${ }^{37,38}$.

For this work, Chitosan has a variable inhibitory effect on biofilm formation regarding different bacterial species. These findings are in agreement with Polyudova et al. who found that Chitosan has a fourfold inhibition of Mycobacterium smegmatis biofilm while has a minimal effect on E.coli strains, he refer his foundation to the increase in the hydrophobicity of attachment surfaces that will decrease the effect of chitosan as a biofilm inhibitor ${ }^{39}$. Puga and his colleagues suggest that environmental stress leads to increased tolerance to Chitosan effect ${ }^{40}$. Also, molecular weight and acetylation degree may control the effectiveness of Chitosan ${ }^{41}$.

Pluronic F127 shows potent antibiofilm activity at low concentration $(1.25 \mathrm{mg} / \mathrm{mL})$, its activity reaches up to $80 \%$ inhibition for strong biofilm producing strains. Treter et al. show similar effect of Pluronic F127 on Staphylococcus epidermidis, it inhibits $90 \%$ of biofilm formation ${ }^{42}$. Although, combination of Pluronic with Chitosan has a similar effect like Pluronic alone, but Pluronic can increase release, solubility and bioavailability of Chitosan. Alvarado-Gomez et al. reported synergistic activity of Pluronic F127 and silver nanoparticles in hydrogel form against biofilm forming Pseudomonas spp and Staphylococcus $s p p^{43}$. Another study by Manaspon et al. reported increased cytotoxic activity of doxorubicin against breast cancer cells using folate-conjugated pluronic F127/chitosan core-shell nanoparticles ${ }^{18}$. From our work and previous studies, we conclude that Pluronic F 127 can increase effect of Chitosan suggesting their use in combination at low concentration level with high efficacy against biofilm formation.

\section{CONCLUSION}

Nosocomial infection represents a substantial health problem. A significant number of nosocomial isolates were moderate to strong biofilm producers. Despite of finding a new effective antibiotic for treatment of highly resistant organisms, inhibition of transmission can represent a new effective approach. Chitosan and Pluronic F127 are safe biocompatible agents used in different medical formulation. They show a potential degree of biofilm inhibition. They could be used as an alternative source for inhibition of bacterial resistance and transmission.

\section{ACKNOWLEDGMENTS}

We acknowledge Dr. Mohammed Ragab (Pharmacology Department, Faculty of Pharmacy, Beni-Suef University) for performing statistical analysis using SPSS software.

\section{AUTHORS' CONTRIBUTION}

AFA and WGH design the experiment; $D E, A F A$ performed the experiments; WGH, AFA, OMS analyzed the data; AFA, OMS write the original draft. All authors read and approved the manuscript.

\section{FUNDING}

None.

\section{CONFLICT OF INTEREST}

The authors declare that there is no conflict of interest.

\section{ETHICS STATEMENT}

The study was approved by Ethics Committee, Faculty of Medicine, Beni-Suef University.

\section{DATA AVAILABILITY}

All datasets generated or analyzed during this study are included in the manuscript.

\section{REFERENCES}

1. Edwardson S, Cairns C. Nosocomial infections in the ICU. Anaesthesia \& Intensive Care Medicine. 2019;20(1):14-18. doi: 10.1016/j.mpaic.2018.11.004

2. Jenkins DR. Nosocomial infections and infection control. Medicine. 2017;45(10):629-633. doi: 10.1016/j.mpmed.2017.07.005

3. Jamal M, Ahmad W, Andleeb S, et al. Bacterial biofilm and associated infections. Journal of the Chinese Medical Association. 2018;81(1):7-11. doi: 10.1016/j. jcma.2017.07.012

4. Sutherland IW. Polysaccharases for microbial exopolysaccharides. Carbohydrate Polymers. 1999;38(4):319-328. doi: 10.1016/S01448617(98)00114-3

5. Donlan RM, Costerton JW. Biofilms: survival 
mechanisms of clinically relevant microorganisms. Clin Microbiol Rev. 2002;15(2):167-193. doi: 10.1128/ CMR.15.2.167-193.2002

6. Donlan RM. Biofilms: microbial life on surfaces. Emerg Infect Dis. 2002;8(9):881-890. doi: 10.3201/ eid0809.020063

7. Hoyle BD, Costerton JW. Bacterial resistance to antibiotics: the role of biofilms. Progress in drug Research Fortschritte der Arzneimittelforschung Progres des Recherches Pharmaceutiques. 1991;37:91105. doi: 10.1007/978-3-0348-7139-6_2

8. Cerqueira GM, Peleg AY. Insights into Acinetobacter baumannii pathogenicity. IUBMB Life. 2011;63(12):1055-1060. doi: 10.1002/iub.533

9. Molina-Manso D, Del-Prado G, Gomez-Barrena E, Cordero-Ampuero J, Fernandez-Roblas R, Esteban J. Effect of different agents with potential antibiofilm activity on antimicrobial susceptibility of biofilms formed by Staphylococcus spp. isolated from implant-related infections. The Journal Of Antibiotics. 2016;69:686-688. doi: 10.1038/ja.2016.9

10. Algburi A, Comito N, Kashtanov D, Dicks LMT, Chikindas ML. Control of Biofilm Formation: Antibiotics and Beyond. Appl Environ Microbiol. 2017;83(3):e0250802516. doi: 10.1128/AEM.02508-16

11. Rossi LM, Rangasamy P, Zhang J, Qiu XQ, Wu GY. Research advances in the development of peptide antibiotics. J Pharm Sci. 2008;97(3):1060-1070. doi: 10.1002/jps.21053

12. Eckhart L, Fischer $\mathrm{H}$, Barken KB, Tolker-Nielsen $\mathrm{T}$, Tschachler E. DNase1L2 suppresses biofilm formation by Pseudomonas aeruginosa and Staphylococcus aureus. Br J Dermatol. 2007;156(6):1342-1345. doi: 10.1111/j.1365-2133.2007.07886.x

13. Kalpana BJ, Aarthy S, Pandian SK. Antibiofilm activity of alpha-amylase from Bacillus subtilis S8-18 against biofilm forming human bacterial pathogens. Appl Biochem Biotechnol. 2012;167(6):1778-1794. doi: 10.1007/s12010-011-9526-2

14. Uroz S, Dessaux Y, Oger P. Quorum Sensing and Quorum Quenching: The Yin and Yang of Bacterial Communication. Chem BioChem. 2009;10(2):205-216. doi: 10.1002/cbic.200800521

15. Huh AJ, Kwon YJ. "Nanoantibiotics": A new paradigm for treating infectious diseases using nanomaterials in the antibiotics resistant era. $J$ Control Release. 2011;156(2):128-145. doi: 10.1016/j. jconrel.2011.07.002

16. Goy RC, de Britto D, Assis OBG. A review of the antimicrobial activity of chitosan. Polimeros. 2009;19(3):241-247. doi: 10.1590/S010414282009000300013

17. Romic MD, Klaric MS, Lovric J, et al. Melatonin-loaded chitosan/Pluronic ${ }^{\circledR}$ F127 microspheres as in situ forming hydrogel: An innovative antimicrobial wound dressing. Eur J Pharm Biopharm. 2016;107:67-79. doi: 10.1016/j.ejpb.2016.06.013

18. Manaspon C, Viravaidya-Pasuwat K, Pimpha N. Preparation of Folate-Conjugated Pluronic F127/ Chitosan Core-Shell Nanoparticles Encapsulating Doxorubicin for Breast Cancer Treatment. Journal of Nanomaterials. 2012;2012:1-11. doi:

\section{$10.1155 / 2012 / 593878$}

19. Hosseinzadeh H, Atyabi F, Dinarvand R, Ostad SN. Chitosan-Pluronic nanoparticles as oral delivery of anticancer gemcitabine: preparation and in vitro study. Int J Nanomedicine. 2012;7:1851-1863. doi: 10.2147/ IJN.S26365

20. Escalona-Rayo CF, Serrano-Castaneda P, LopezCervantes M, Escobar-Chavez JJ. Optimization of Unidirectional Mucoadhesive Buccal Patches Based on Chitosan and Pluronic ${ }^{\circledR}$ F-127 for Metoprolol Controlled Release: In Vitro and Ex Vivo Evaluations. Journal of Pharmaceutical Innovation. 2019. doi: 10.1007/s12247-019-09401-8

21. Pepic I, Hafner A, Lovric J, Pirkic B, Filipovic-Grccic J. A Nonionic Surfactant/Chitosan Micelle System in an Innovative Eye Drop Formulation. Journal of Pharmaceutical Sciences. 2010;99(10):4317-4325. doi: 10.1002/jps.22137

22. CLSI. Performance Standards for Antimicrobial Susceptibility Testing. 26th ed. CLSI supplement M100S ed. Wayne, PA: Clinical and Laboratory Standards Institute; 2016.

23. Stepanovic S, Vukovic D, Dakic I, Savic B, SvabicVlahovic M. A modified microtiter-plate test for quantification of staphylococcal biofilm formation. $J$ Microbiol Methods. 2000;40(2):175-179. doi: 10.1016/ S0167-7012(00)00122-6

24. Pierce CG, Uppuluri P, Tristan AR, et al. A simple and reproducible 96 -well plate-based method for the formation of fungal biofilms and its application to antifungal susceptibility testing. Nature Protocols. 2008;3(9):1494-1500. doi: 10.1038/nprot.2008.141

25. Gomes LC, Mergulhao FJ. SEM Analysis of Surface Impact on Biofilm Antibiotic Treatment. Scanning. 2017;2017:2960194. doi: 10.1155/2017/2960194

26. Mama M, Abdissa A, Sewunet T. Antimicrobial susceptibility pattern of bacterial isolates from wound infection and their sensitivity to alternative topical agents at Jimma University Specialized Hospital, South-West Ethiopia. Ann Clin Microbiol Antimicrob. 2014;13(14). doi: 10.1186/1476-0711-13-14

27. Dessie W, Mulugeta G, Fentaw S, Mihret A, Hassen $M$, Abebe E. Pattern of Bacterial Pathogens and Their Susceptibility Isolated from Surgical Site Infections at Selected Referral Hospitals, Addis Ababa, Ethiopia. Int J Microbiol. 2016;2016:2418902-2418902. doi: $10.1155 / 2016 / 2418902$

28. Feleke T, Eshetie S, Dagnew M, et al. Multidrugresistant bacterial isolates from patients suspected of nosocomial infections at the University of Gondar Comprehensive Specialized Hospital, Northwest Ethiopia. BMC Research Notes. 2018;11(1):602-602. doi: 10.1186/s13104-018-3709-7

29. Azene MK, Beyene BA. Bacteriology and antibiogram of pathogens from wound infections at Dessie Laboratory, North-east Ethiopia. Tanzania Journal of Health Research. 2011;13(4):68-74. doi: 10.4314/thrb. v13i4.64901

30. Matta R, Hallit S, Hallit R, Bawab W, Rogues A-M, Salameh P. Epidemiology and microbiological profile comparison between community and hospital acquired infections: A multicenter retrospective study 
in Lebanon. Journal of Infection and Public Health. 2018;11(3):405-411. doi: 10.1016/j.jiph.2017.09.005

31. Peters L, Olson L, Khu DTK, et al. Multiple antibiotic resistance as a risk factor for mortality and prolonged hospital stay: A cohort study among neonatal intensive care patients with hospital-acquired infections caused by gram-negative bacteria in Vietnam. PLOS ONE. 2019;14(5):e0215666. doi: 10.1371/journal. pone. 0215666

32. Sserwadda I, Lukenge M, Mwambi B, Mboowa G, Walusimbi A, Segujja F. Microbial contaminants isolated from items and work surfaces in the postoperative ward at Kawolo general hospital, Uganda. BMC Infectious Diseases. 2018;18(1):68. doi: 10.1186/ s12879-018-2980-5

33. Kassam NA, Damian DJ, Kajeguka D, Nyombi B, Kibiki GS. Spectrum and antibiogram of bacteria isolated from patients presenting with infected wounds in a Tertiary Hospital, northern Tanzania. BMC Research Notes. 2017;10(1):757. doi: 10.1186/s13104-0173092-9

34. Mauldin PD, Salgado CD, Hansen IS, Durup DT, Bosso JA. Attributable Hospital Cost and Length of Stay Associated with Health Care-Associated Infections Caused by Antibiotic-Resistant GramNegative Bacteria. Antimicrob Agents Chemother. 2010;54(1):109. doi: 10.1128/AAC.01041-09

35. Mulla S, Revdiwala S. Assessment of biofilm formation in device-associated clinical bacterial isolates in a tertiary level hospital. Indian J Pathol Microbiol. 2011;54(3):561-564. doi: 10.4103/0377-4929.85093

36. Singhai M, Malik A, Shahid M, Malik MA, Goyal R. A study on device-related infections with special reference to biofilm production and antibiotic resistance. J Glob Infect Dis. 2012;4(4):193-198. doi:
10.4103/0974-777X.103896

37. Yang D, Zhang Z. Biofilm-forming Klebsiella pneumoniae strains have greater likelihood of producing extendedspectrum $\beta$-lactamases. J Hosp Infect. 2008;68(4):369371. doi: 10.1016/j.jhin.2008.02.001

38. Vuotto C, Longo F, Balice MP, Donelli G, Varaldo PE. Antibiotic Resistance Related to Biofilm Formation in Klebsiella pneumoniae. Pathogens. 2014;3(3):743-758. doi: 10.3390/pathogens 3030743

39. Polyudova TV, Shagdarova BT, Korobov VP, Varlamov VP. Bacterial Adhesion and Biofilm Formation in the Presence of Chitosan and Its Derivatives. Microbiology. 2019;88(2):125-131. doi: 10.1134/ S0026261719020085

40. Puga $\mathrm{CH}$, SanJose C, Orgaz B. Biofilm development at low temperatures enhances Listeria monocytogenes resistance to chitosan. Food Control. 2016;65:143-151. doi: 10.1016/j.foodcont.2016.01.012

41. Mu H, Zhang A, Zhang L, Niu H, Duan J. Inhibitory effects of chitosan in combination with antibiotics on Listeria monocytogenes biofilm. Food Control. 2014;38:215220. doi: 10.1016/j.foodcont.2013.10.032

42. Treter J, Bonatto F, Krug C, Soares GV, Baumvol IJR, Macedo AJ. Washing-resistant surfactant coated surface is able to inhibit pathogenic bacteria adhesion. Appl Surf Sci. 2014;303:147-154. doi: 10.1016/j. apsusc.2014.02.123

43. Alvarado-Gomez E, Martinez-Castanon G, SanchezSanchez R, Ganem-Rondero A, Yacaman MJ, MartinezGutierrez F. Evaluation of anti-biofilm and cytotoxic effect of a gel formulation with Pluronic F-127 and silver nanoparticles as a potential treatment for skin wounds. Materials Science and Engineering: $C$. 2018;92:621-630. doi: 10.1016/j.msec.2018.07.023 